\title{
Burnout y empatía en cuidadores profesionales de personas mayores
}

\author{
Juan P. Martínez, Inmaculada Méndez y Julia García-Sevilla \\ Universidad de Murcia (España)
}

La finalidad del presente trabajo ha sido analizar la empatía cognitiva y afectiva en trabajadores profesionales en un centro de personas mayores institucionalizadas. La empatía supone la capacidad de tener en el punto de vista de otros, así como entender sus sentimientos y emociones, tal como postula la Teoría de la Mente. La despersonalización es una acción de alejamiento que actúa de mecanismo de defensa y es típica en el profesional con altos niveles de burnout. Por ello, se ha analizado este síndrome y su relación con el constructo de empatía en 20 cuidadores profesionales. Los instrumentos utilizados han sido el Test de Empatía Cognitiva y Afectiva (TECA, compuesto por las escalas de adopción de perspectivas, comprensión emocional, estrés empático y alegría empática; el Inventario de Burnout de Maslach (MBI, agotamiento emocional, despersonalización y realización profesional) y una encuesta elaborada $a d$ hoc para el personal laboral para la recogida de información sociodemográfica y del puesto de trabajo. Los resultados muestran una correlación significativa positiva, con tamaño del efecto alto, entre la edad y el estrés empático y entre éste y el agotamiento emocional. También se ha encontrado significación positiva entre despersonalización y alegría empática. Las conclusiones de esta investigación conllevan una reflexión acerca de la configuración y estructura definitoria de este mecanismo de defensa, así como de su relación con la empatía en el ámbito laboral.

Palabras clave: Empatía, burnout, despersonalización, estrés, cuidador profesional.

Burnout and empathy among professional caregivers of elderly. The aim of this research has been to analyze cognitive and affective empathy among professional workers in an institutionalized center. Empathy is the capacity of taking into account others points of with feelings and emotions as Theory of Mind claims. Depersonalization emerges in caregivers in certain circumstances. This acts as a mechanism of defense and is typical in the professional with high levels of burnout. For this reason, it proceeded to examine this syndrome and its relationship with empathy among 20 professional caregivers. The instruments used were the Cognitive and Affective Empathy Scale (consisted of scales understanding of perspectives, emotional understanding, empathic stress and sympathetic joy), Maslach Burnout Inventory (emotional exhaustion, depersonalization and professional accomplishment) and an ad hoc survey for the workforce for the collection of sociodemographic and workplace information. The results show a significant positive correlation, with high effect size, between age and empathic stress and between this one and emotional exhaustion. Positive significance between depersonalization and sympathetic joy has also been found. The conclusions of this research lead a reflection on the configuration and structure of this defense mechanism and its relationship with empathy in the workplace.

Keywords: Empathy, burnout, depersonalization, stress, professional caregivers.

Correspondencia: Juan P. Martínez. Facultad de Psicología. Departamento de Psicología Evolutiva y de la Educación. Universidad de Murcia. CP 30100. Campus de Espinardo, Murcia (España). E-mail: juanpedromartinezramon@um.es 
La empatía es un concepto incluido dentro de las habilidades sociales y que hace referencia a la capacidad de ponerse bajo el punto de vista de otra persona. Conlleva la capacidad y competencia social de poder "sentir" o hacerse a la idea de la situación que está viviendo o por la que está pasando otra persona. Existe una gran variedad de definiciones del concepto de empatía (Baron-Cohen y Weelwright, 2004). La empatía es un aspecto esencial de las conductas prosociales; éstas son acciones orientadas principalmente a beneficiar a otras personas lo cual incluye compartir, ayudar, entre otros aspectos (Eisenberg, Fabes, y Spinrad, 2006; Mestre, 2014; Richard, 2014). Ésta se transmite tanto mediante mensajes verbales (dar respuestas oportunas, trasmitir un interés por la vida del usuario...) como no verbales (contacto ocular directo, brazos abiertos, entre otros) (Müggenburg, Olvera, Riveros, Hernández-Guillén, y Aldana, 2015). Algunos marcos explicativos, como es el caso de la Teoría de la Mente, han tratado de describir cómo un ser humano es capaz de ponerse en el lugar de otro, es decir, de qué modo puede comprender e incluso predecir la conducta de otras personas aludiendo a aspectos metacognitivos (Tirapu-Ustárroz, Pérez-Sayes, Erekatxo-Bilbao, y Pelegrín-Valero, 2007).

La empatía puede analizarse a su vez a través de otros conceptos como son la adopción de perspectivas, la comprensión emocional, el estrés empático y la alegría empática (López-Pérez, Fernández-Pinto, y Abad, 2008). La adopción de perspectivas hace referencia a la capacidad de ponerse en el lugar de otra persona; la comprensión emocional a la habilidad de comprender las emociones de los demás; el estrés empático se da en aquellas personas que, por ejemplo, sienten tristeza al escuchar malas noticias que afectan a personas desconocidas; y la alegría empática es la capacidad de sentirse bien cuando a otras personas les pasa algo positivo, se trata, por tanto, de la capacidad de poder compartir este tipo de emociones.

Por otro lado, el síndrome de burnout, también denominado Síndrome de Estar Quemado en el Trabajo (SQT), fue propuesto para designar un conjunto de síntomas que se daban en el ámbito laboral y que conllevaba agotamiento, distanciamiento así como bajo rendimiento, por citar unos ejemplos, en el trabajador (Freudenberger, 1974). Aquellas profesiones cuyas funciones exigen un continuo flujo de interacciones con otra persona, con la consiguiente necesidad de adaptación a intereses, preferencias, valores, necesidades... tienen una mayor probabilidad de sufrir el síndrome, tal como ocurre en el caso de los cuidadores profesionales (Martínez, Méndez, Secanilla, Benavente, y García-Sevilla, 2014). El síndrome puede ser descrito como un proceso en el cual el sujeto se encuentra agotado emocionalmente, sintiéndose sin fuerzas para poder desarrollar su actividad laboral con normalidad. Como mecanismo de defensa ante la necesidad de volver a los niveles habituales de energía surge la despersonalización que consiste grosso modo en tratar al usuario, al paciente o al cliente como un objeto y no como un sujeto con una entidad psicológica. Finalmente, la tercera 
dimensión es una baja realización personal en el trabajo (Martínez, 2015; Maslach, 2009). Recientes estudios abogan por la idea de que el agotamiento emocional que es un constructo clave en la formación del síndrome, puede surgir desde el mismo comienzo de la formación universitaria, antes de la incorporación al mundo laboral, en los primeros contactos durante las prácticas (Faye-Dumanget, Abdel-Halim, y Marjolet, 2015). Los cuidadores profesionales de residencias son un colectivo diana para sufrir el síndrome debido a las características de sus funciones, presentando altos niveles de estrés y altas puntuaciones en las dimensiones que conforman el burnout (Méndez, Secanilla, Martínez, y Navarro, 2011; Otero-López, Villardefrancos, Castro, y Santiago, 2014; Gastaldi, Pasta, Longobardi, Prino, y Quaglia, 2014) y en la estabilidad de las puntuaciones en despersonalización transcurrido el tiempo (Martínez, Méndez, Secanilla, y González, 2012). Se trata de un grupo de profesionales expuesto a una gran carga la cual puede provenir de variables muy diversas y cuyo origen se encuentra en la actualidad en proceso de análisis (Crespo y Rivas, 2015). El aumento de la esperanza de vida y la correlación positiva entre edad y prevalencia de trastornos mentales apoya la necesidad de prestar atención a este colectivo profesional (Gázquez, Pérez, Lucas, y Yuste, 2008).

Del análisis teórico de las dimensiones del burnout extraemos que una de sus características es el trato despersonalizado del trabajador hacia el usuario. Se trata, por tanto, de un bloque de las habilidades sociales y de las capacidades empáticas para evitar sufrir un agotamiento emocional. No obstante, el mismo mecanismo que se pone en marcha para aliviar tensiones es precisamente el que ejerce un efecto negativo y favorece junto a otras dimensiones ya mencionadas la aparición del síndrome.

Por la relación que puede guardar el concepto de empatía con el síndrome de burnout, el objetivo general de este trabajo ha sido analizar la empatía cognitiva y afectiva en trabajadores profesionales en un centro de personas mayores institucionalizadas. De dicha finalidad se desprenden los siguientes objetivos específicos: (a) estudiar la relación entre el constructo de empatía y diversas variables sociodemográficas y (b) examinar la relación existente entre la empatía y las dimensiones del síndrome de burnout.

\section{MÉTODO}

\section{Participantes}

Se ha llevado a cabo la aplicación de varios instrumentos de medición psicológica a 20 cuidadores profesionales que ejercen sus funciones en una residencia en Lorca (Murcia, España) de un total de 44 profesionales que desempeñan sus funciones en ella. El $22.2 \%$ son varones y el $77.8 \%$ mujeres. La nacionalidad del $100 \%$ de los sujetos es española. La edad de los trabajadores oscila en un amplio intervalo que va de 
los 22 a los 62 años. El 29.6\% de la muestra está casada. Aproximadamente la mitad de los participantes tienen un contrato fijo (55\%), turno de mañana (55\%) y/o estudios universitarios $(50 \%)$.

\section{Procedimiento}

El diseño de esta investigación está basado en un enfoque no experimental de tipo comparativo. Con respecto al procedimiento, se llevó a cabo una entrevista en una residencia sita en el municipio de Lorca (Región de Murcia). Se informó de los objetivos a la dirección, se solicitó el consentimiento y se procedió a realizar una segunda información a los sujetos participantes, formando parte del estudio siempre de forma voluntaria y asegurándose la confidencialidad y el anonimato. Los cuestionarios fueron administrados por los propios autores del estudio durante los meses de marzo y abril de 2014. Tras la recopilación de los datos, se realizó el análisis descriptivo e inferencial en el programa estadístico SPSS (v. 19).

\section{Instrumentos}

Se han utilizado los siguientes instrumentos de medición psicológica, los cuales, fueron administrados a los cuidadores profesionales. Más concretamente, se pasó el Test de Empatía Cognitiva y Afectiva (TECA) (López-Pérez et al., 2008) cuyo objetivo es la apreciación de la capacidad empática bajo una perspectiva cognitiva y afectiva. El test está compuesto por las escalas de adopción de perspectivas, comprensión emocional, estrés empático y alegría empática. Sus 33 elementos permiten predecir la empatía de un sujeto ante una situación emocional compleja. El cuestionario tiene utilidad tanto dentro del ámbito organizacional como del social y del clínico. Presenta una consistencia interna de .86. El presente estudio obtuvo un índice alfa de Cronbach de .082 .

También se administró el Inventario de Burnout de Maslach (MBI) (Maslach y Jackson, 1986). Se basa en la existencia de tres dimensiones, en concreto, agotamiento emocional (AE), despersonalización (DP) y realización personal en el trabajo (RP), fruto del análisis factorial de 22 ítems. Alfa de Cronbach presenta una oscilación de .71 a .90 (Alarcón, Vaz, y Guisado, 2002). En este estudio obtuvo un índice de fiabilidad de Cronbach cercano a .60.

Por otro lado, se diseñó una encuesta elaborada ad hoc para el personal laboral destacando variables sociodemográficas tales como sexo, edad, estado civil, así como características del puesto de trabajo y referente a la formación previa. 


\section{RESULTADOS}

Con relación al análisis de las dimensiones que componen el constructo de empatía, los resultados muestran una puntuación media más alta en la dimensión comprensión empática ( $M=57.35, D T=27.74)$ en el cuestionario TECA en comparación con el resto de dimensiones de dicha prueba (Tabla 1).

\begin{tabular}{lccccc}
\multicolumn{6}{c}{ Tabla 1. Índices de dispersión $(M$ y $D T)$ de las dimensiones del cuestionario TECA (empatía) } \\
\hline & $\begin{array}{c}\text { Adopción de } \\
\text { perspectivas }\end{array}$ & $\begin{array}{c}\text { Comprensión } \\
\text { empática }\end{array}$ & $\begin{array}{c}\text { Estrés } \\
\text { empático }\end{array}$ & $\begin{array}{c}\text { Alegría } \\
\text { empática }\end{array}$ & Teca Total \\
\hline Media & 41.35 & 57.35 & 39.45 & 47.00 & 112.95 \\
\hline Desv. Típica & 20.69 & 27.74 & 25.31 & 22.39 & 13.76 \\
\hline
\end{tabular}

Del análisis de las variables sociodemográficas y de las dimensiones de empatía estudiadas aplicando la Correlación de Pearson se obtiene una relación significativa positiva, con tamaño del efecto alto, entre la edad y el estrés empático $\left(r_{p}=.591, p=.006\right)$ (Tabla 2).

Tabla 2. Matriz de correlaciones entre las dimensiones del síndrome de burnout, empatía y edad

\begin{tabular}{|c|c|c|c|c|c|c|c|c|c|}
\hline & Edad & $\mathrm{AE}$ & DP & $\mathrm{RP}$ & APersp & CEmp & EEmp & AEmp & TT \\
\hline Edad & 1 & & & & & & & & \\
\hline $\mathrm{AE}$ & .313 & 1 & & & & & & & \\
\hline DP & .403 & .860 & 1 & & & & & & \\
\hline RP & -.370 & -.183 & -.043 & 1 & & & & & \\
\hline AP & .068 & .061 & .176 & -.045 & 1 & & & & \\
\hline CEmp & .176 & .256 & .357 & -.161 & $.768^{* *}$ & 1 & & & \\
\hline EEmp & $.591 * *$ & $.511 *$ & .418 & -.272 & $.521 *$ & .437 & 1 & & \\
\hline AEmp & .232 & -.234 & $.509 *$ & -.058 & $.671 * *$ & $.608 * *$ & .337 & 1 & \\
\hline TT & .338 & .338 & $.553 *$ & -.172 & $.896 * *$ & $.872 * *$ & $.711 * *$ & $.787 * *$ & 1 \\
\hline
\end{tabular}

Tal como cabría esperar, se obtuvo una correlación significativa positiva y con tamaño del efecto alto entre la mayoría de las dimensiones que componen el constructo de empatía: comprensión empática-adopción de perspectivas $\left(r_{p}=.768\right.$, $p=.000)$, estrés empático- adopción de perspectivas $\left(r_{p}=.521, p=.018\right)$, alegría empática-adopción de perspectivas $\left(r_{p}=.671, p=.001\right)$, Teca Total-adopción de perspectivas $\left(r_{p}=.896, p=.000\right)$, alegría empática-comprensión empática $\left(r_{p}=.608\right.$, $p=.004)$, Teca Total-comprensión empática $\left(r_{p}=.872, p=.000\right)$ así como Teca Total-estrés empático $\left(r_{p}=.711, p=.000\right)$, Teca Total-alegría empática $\left(r_{p}=.787, p=.000\right)$.

Más variada es la relación existente entre las dimensiones del síndrome de estar quemado en el trabajo con el constructo de empatía y de sus respectivas dimensiones, al hallarse una correlación significativa positiva, con tamaño del efecto 
alto, entre el agotamiento emocional y el estrés empático $\left(r_{p}=.511, p=.021\right)$ (Figura 1). De modo similar, se encuentra una correlación significativa positiva, con tamaño del efecto alto, entre la dimensión de despersonalización y la de alegría empatía $\left(r_{p}=.509\right.$, $p=.022$ ) (Figura 2).

Figura 1. Diagrama de dispersión de las variables agotamiento emocional y estrés empático

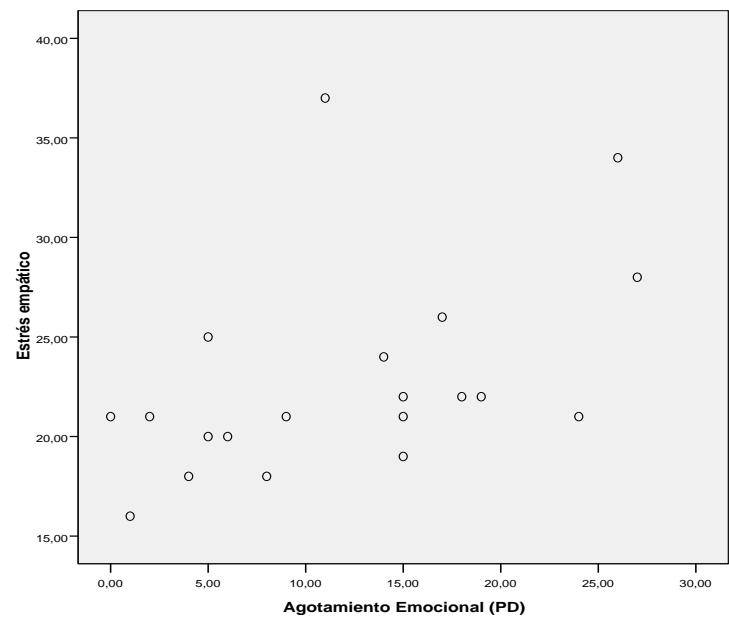

Figura 2. Diagrama de dispersión de las variables despersonalización y estrés empático

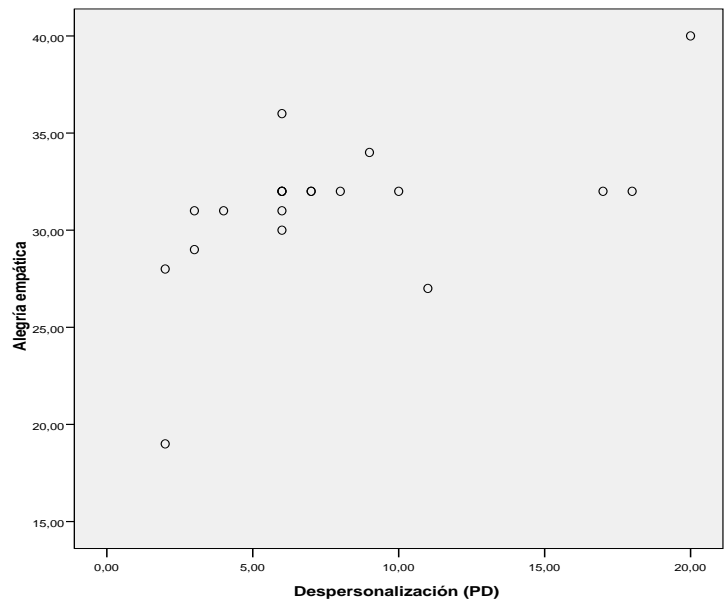




\section{CONCLUSIONES Y DISCUSIÓN}

Las conclusiones que se extraen de este estudio son: la existencia de una correlación estadísticamente significativa, con tamaño del efecto alto, entre la edad del cuidador profesional de la residencia de Lorca (Murcia) y el estrés empático. Del mismo modo, también se encuentra una correlación similar entre la presencia de estrés empático y agotamiento emocional en el trabajador. También se ha hallado una correlación positiva significativa entre la dimensión de burnout de despersonalización y alegría empática. Estos datos resultan relevantes para comprender cómo se configura el síndrome de estar quemado y arrojan luz acerca de cómo el uso de ciertas estrategias de afrontamiento, probablemente como mecanismo de defensa, pueden resultar disfuncionales al relacionarse con la presencia de despersonalización. Así, aquellos cuidadores profesionales que presentan mayor alegría empática también presentan despersonalización por lo que podemos concluir que aquellas personas que presentan dicha alegría son más vulnerables al estrés laboral y/o a presentar la citada dimensión del burnout. Si bien la relación entre el estrés empático y el agotamiento emocional podían ser predichos, no ha ocurrido lo mismo con la alegría empática y la despersonalización. Los trabajadores con alegría empática sienten felicidad cuando a otras personas les ocurren hechos positivos y alegres en sus vidas $\mathrm{y}$, sin embargo, esta variable correlaciona positivamente con el hecho de alejarse de los usuarios psicológicamente.

Con relación a la vertiente aplicada se debe instruir en formas adecuadas de funcionamiento personal y laboral partiendo de la base de que la situación organización puede ser vista como un entramado de relaciones sociales. En esta línea, las relaciones interpersonales positivas que mantienen los usuarios con los trabajadores y viceversa se basan en la comunicación funcional y en la puesta en marcha de una serie de habilidades sociales. Éstas pueden ser enseñadas y aprendidas a través de programas estructurados de entrenamiento. Éstas son esenciales para la intervención terapéutica (Müggenburg et al., 2015). De aquí se deriva la relevancia de fomentar el diseño y puesta en marcha de programas que consideren el desarrollo de la empatía y de las habilidades sociales en general para mejorar el tratamiento que se le dé al usuario o al paciente. Por tanto, es necesario invertir en formación en el trabajador (Gázquez et al., 2009).

No hay duda de que la empatía es un acto complicado que engloba diversos procesos como la interpretación de las emociones sociales o la cognición social (Tirapu-Ustárroz et al., 2007), aspectos muy relevantes en el ámbito organizacional.

Los programas deberían incorporarse antes de la inclusión del profesional en el ámbito laboral, implantándose desde el mismo momento de la formación universitaria ya que el estudiante necesita desarrollar estrategias frente al estrés desde las fases más iniciales, en sus primeros contactos (Faye-Dumanget et al., 2015). 
Como limitaciones del estudio y futuras propuestas de investigación, se debe indicar la necesidad de aumentar el tamaño muestral en aras de poder generalizar los resultados y de profundizar en la compleja relación que existe entre factores como la despersonalización y el uso de la empatía. En la línea de lo investigado por Crespo y Rivas (2015), también puede resultar interesante indagar en cuáles son las variables que influyen directamente sobre la carga a la que está sometido el cuidador profesional introduciendo nuevos cuestionarios para definir en mayor grado el estado de la cuestión, considerando variables como la resiliencia (Méndez et al, 2015), factores de estrés (Lanzón y Díaz, 2014) así como la incorporación de las estrategias de afrontamiento utilizadas por el sujeto, tal como sea hecho en otros ámbitos de estudio (Martínez, 2015).

\section{REFERENCIAS}

Alarcón, J., Vaz, F., y Guisado, J. (2001). Análisis del síndrome de burnout: psicopatología, estilos de afrontamiento y clima social. Revista de Psiquiatría de la Facultad de Medicina Barna, 28(6), 358-381.

Baron-Cohen, S., y Weelwright, S. (2004). The Empathy Quotient: An Investigation of Adults with Asperger Syndrome or High Functioning Autism, and Normal Sex Differences. Journal of Autism and Developmental Disorders, 34(2), 163-175. doi: 10.1023/ B:JADD.0000022607.19833.00

Crespo, M., y Rivas, M.T. (2015). La evaluación de la carga del cuidador: una revisión más allá de la escala de Zarit. Clínica y Salud, 26(1), 9-15. doi: 10.1016/j.clysa.2014.07.002

Eisenberg, N., Fabes, R.A., y Spinrad, T.L. (2006). Prosocial development. En N. Eisenberg, W. Damon, y R.M. Lerner. Handbook of Child Psychology: Social, Emotional, and Personality, Development, vol.3 (6th ed.) (pp. 646-718). Hoboken, NJ, EUA: John Wiley \& Sons Inc.

Faye-Dumanget, C., Abdel-Halim, B., y Marjolet, M. (2015). Efecto del burnout y del stress posttraumatizante en la empatía en el caso de los estudiantes educadores y enfermeros. Revista Electrónica Medicina, Salud y Sociedad, 5(2), 83-93.

Freudenberger, H. (1974). Staff burn-out. Journal of Social Issues, 30(1), 159-165. doi: 10.1111/j.1540-4560.1974.tb00706.x

Gastaldi, F.G.M., Pasta, T., Longobardi, C., Prino L.E., y Quaglia, R. (2014). Measuring the influence of stress and burnout in teacher-child relationship. European Journal of Education and Psychology, 7(1), 17-28.

Gázquez, J.J., Pérez-Fuentes, M.C., Fernández, M., González, L., Ruiz, I., y Díaz, A. (2009).Oldage stereotypes to the gerontology education: an intergenerational study. European Journal of Education and Psychology, 2(3), 263-273.

Gázquez, J.J., Pérez-Fuentes, M.C., Lucas, F., y Yuste, N. (2008). Prevalencia de los trastornos mentales en la población mayor. Anales de Psicología, 24(2), 327-333. Recuperado de $\mathrm{http} / / / \mathrm{www} . u m . e s / a n a l e s p s / \mathrm{v} 24 / \mathrm{v} 24 \_2 / 16-24 \_2 . p d f$

Lanzón, T., y Díaz, A. (2014). Predictive role of caregiver type, dependency level and time caring on the impact of caring for a dependent relative as a stressor. European Journal of Investigation in Health, Psychology and Education, 4(3), 193-202.

López-Pérez, B., Fernández-Pinto, I., y Abad, F.J. (2008). TECA. Test de Empatía Cognitiva y Afectiva. Madrid: Tea Ediciones, S.A. 
Martínez, J.P. (2015). Cómo se defiende el profesorado de secundaria del estrés: burnout y estrategias de afrontamiento. Journal of Work and Organizational Psychology, 31, 1-9. doi: 10.1016/j.rpto.2015.02.001

Martínez, J.P., Méndez, I., Secanilla, E., y González, E. (2012). Evolución de los niveles de burnout en un estudio comparativo en cuidadores profesionales tras una situación de estrés postraumático. European Journal of Investigation in Health, Psychology and Education, 2(1), 29-39. doi: 10.1989/ejihpe.v2il.12

Martínez, J.P., Méndez, I., Secanilla, E., Benavente, A., y García-Sevilla, J. (2014). Burnout en cuidadores profesionales y calidad de vida en residentes de centros institucionalizados. European Journal of Investigation in Health, Psychology and Education, 4(1), 41-53. doi: 10.1989/ejihpe.v4i1.39

Maslach, C. (2009). Comprendiendo el burnout. Ciencia y Trabajo, 11(32), 37-43.

Maslach, C., y Jackson, S.E. (1986). Maslach Burnout Inventory. Palo Alto, CA: Consulting Psychologists Press. Recuperado de http://www.vitoria-gasteiz.org/wb021/http/ contenidosEstaticos/adjuntos/es/16/40/51640.pdf

Méndez, I., García-Sevilla, J., Martínez, J.P., Boti, M.A., Cánovas, A.B., y Clemente, Y. (2015). Resiliencia en trabajadores y en residentes de un centro de personas mayores institucionalizadas. European Journal of Investigation in Health, Psychology and Education, 5(1), 65-73. doi: 10.1989/ejihpe.v1i1.91

Méndez, I., Secanilla, E., Martínez, J.P., y Navarro, J. (2011). Estudio comparativo de burnout en cuidadores profesionales de personas mayores institucionalizadas con demencias y otras enfermedades. European Journal of Investigation in Health, Psychology and Education, 1(2), 61-70. doi: 10.1989/ejihpe.v1i2.5

Mestre, M.V. (2014). Desarrollo prosocial: crianza y escuela. Revista Mexicana de Investigación en Psicología, 6(2), 115-134. Recuperado de http://www.revistamexicana deinvestigacionenpsicologia.com/articulos/render/150/1

Müggenburg, C., Olvera, S., Riveros, A., Hernández-Guillén, C., y Aldana, A. (2015). Autoevaluación de enfermeras respecto a la comunicación percibida con pacientes como resultado de un entrenamiento. Enfermería Universitaria, 12(1), 12-18. doi: 10.1016/j.reu.2014.10.001

Otero-López, J.M., Villardefrancos, E., Castro, C., y Santiago, M.J. (2014). Stress, positive personal variables and burnout: A path analytic approach. European Journal of Education and Psychology, 7(2), 95-106.

Richaud, M.C. (2014). Algunos aportes sobre la importancia de la empatía y la prosocialidad en el desarrollo humano. Revista Mexicana de Investigación en Psicología, 6(2), 171-176.

Tirapu-Ustárroz, J., Pérez-Sayes, G., Erekatxo-Bilbao, M., y Pelegrín-Valero, C. (2007). ¿Qué es la teoría de la mente? Revista de Neurología, 44(8), 479-489.

Recibido: 1 de abril de 2015

Recepción Modificaciones: 1 de septiembre de 2015

Aceptado: 15 de noviembre de 2015 